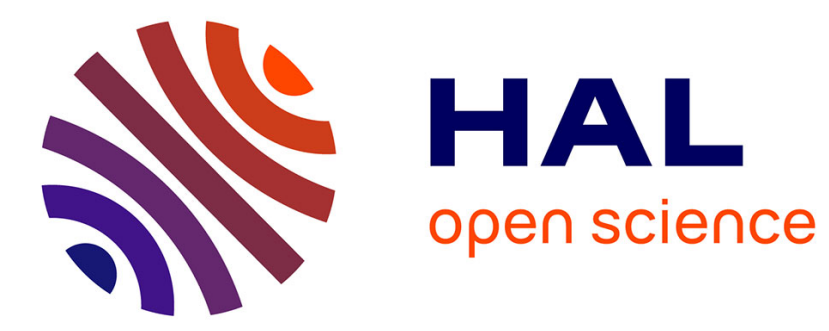

\title{
Numerical assessment of the macroscopic strength criterion of reinforced soils using semidefinite programming
}

\author{
Maxime Gueguin, Ghazi Hassen, Patrick de Buhan
}

\section{To cite this version:}

Maxime Gueguin, Ghazi Hassen, Patrick de Buhan. Numerical assessment of the macroscopic strength criterion of reinforced soils using semidefinite programming. International Journal for Numerical Methods in Engineering, 2014, 99 (7), pp.522-541. 10.1002/nme.4716 . hal-01052946

\section{HAL Id: hal-01052946 \\ https: / hal-enpc.archives-ouvertes.fr/hal-01052946}

Submitted on 29 Jul 2014

HAL is a multi-disciplinary open access archive for the deposit and dissemination of scientific research documents, whether they are published or not. The documents may come from teaching and research institutions in France or abroad, or from public or private research centers.
L'archive ouverte pluridisciplinaire HAL, est destinée au dépôt et à la diffusion de documents scientifiques de niveau recherche, publiés ou non, émanant des établissements d'enseignement et de recherche français ou étrangers, des laboratoires publics ou privés. 


\title{
Numerical assessment of the macroscopic strength criterion of reinforced soils, using semidefinite programming
}

\author{
Maxime Gueguin*, Ghazi Hassen, Patrick de Buhan \\ Université Paris-Est, Laboratoire Navier (UMR 8205), CNRS, ENPC, IFSTTAR, 6 et 8 \\ av. B. Pascal, 77455 Marne-La-Vallée, France
}

\begin{abstract}
The macroscopic strength properties of reinforced soils, regarded as periodic composite materials, are investigated by means of a fem-based formulation of both the static and kinematic approaches of yield design applied to the reinforced soil's unit cell. Since the reinforced soil's individual constituents obey a 3D Mohr-Coulomb strength condition, such a numerical problem can be treated trough an optimization procedure using semidefinite programming. The whole numerical procedure is applied to the derivation of both lower bound and upper bound estimates to the macroscopic yield surface of a soil reinforced either by columnar inclusions (stone columns) or a double array of trenches (cross trench reinforcement). The so-obtained results highlight the efficiency of the proposed numerical method.
\end{abstract}

Keywords: yield design homogenization theory, semidefinite programming, generalized plane strain, stone columns, cross trench reinforced soil

\section{Introduction}

Analysing the load carrying of materials and structures in the framework of yield design (or limit analysis) theory has been an important subject of research for decades and was firstly performed with analytical tools. It has known a significant progress with the development of computing capacities. Specifically developed numerical tools have helped to treat more complex problems and represent a valuable complement for the analytical studies. In this context, both static and kinematic approaches of yield design theory may be formulated as optimization problems and can be solved by discretizing the problem into finite elements.

The first proposed formulations have consisted in linearizing the classical criteria (von Mises, Drucker-Prager, Mohr-Coulomb in 2D) and employing linear

\footnotetext{
${ }^{*}$ Corresponding author

Email address: maxime.gueguin@enpc.fr (Maxime Gueguin)
} 
programming (LP) to solve the optimization problem resulting therefrom [1-4]. Seeking to improve the approximation of those criteria, they have been directly expressed as non-linear yield functions and problems have been optimized using non-linear programming (NLP) [5-7].

Meanwhile, the improvement of available optimization codes in the last 10 years has brought new opportunities for the numerical limit analysis. The possibility to use second-order conic programming (SOCP) has allowed to take into account the real yield function of materials obeying a Drucker-Prager (and by the way von Mises) strength criterion, since it rigorously belongs to this set of cones. Many articles report the use of the SOCP to solve limit analysis problems with various optimization softwares [8-14].

Among the latest subjects of research, was the possibility of implementing such optimization procedures to the general, but more difficult, situation of problems involving materials which obey a 3D Mohr-Coulomb strength criterion. Indeed, in such a case, the corresponding yield function can no longer be considered as a second-order conic condition, since its definition depends on positive semidefinite specific tensors. New formulations of such a strength criterion, as well as its support function, have been quite recently developed and applications have been made using semidefinite programming (SDP) $[11,15,16]$. These formulations allow to take the Mohr-Coulomb and Tresca criteria in numerical limit analysis into account in a rigorous way.

A quite significant field of application of the yield design approach, is the determination of the macroscopic strength properties of composite materials, viewed as periodic heterogeneous media, which can be treated in the context of the yield design homogenization method. Applications of this method can be found in a general framework [17] or in the context of reinforced soil mechanics [18]. So far, the macroscopic strength properties of composite materials were determined using analytical methods $[19,20]$ or numerical elastoplastic procedures $[21,22]$. While the opportunity to use numerical limit analysis methods has rarely been explored for this kind of composite material [23], SOCP or SDP formulations have never been implemented for such applications.

This contribution outlines the static and kinematic approaches of yield design homogenization theory with a specific formulation for a composite material (section 2). The formulation of this problem as an optimization problem using SDP is given. Section 3 describes the study of composites reinforced by long fibers, introducing the generalized plane strain conditions. In section 4, the homemade limit analysis code will be described in details, in the context of generalized plane strain conditions. Implementation is developed for obtaining a lower bound estimate to the macroscopic strength domain, as well as an upper bound estimate. The numerical procedure is then applied to the case of a stone column reinforced soil (section 5) and a cross trench reinforced soil (section 6). The performance and the calculation time of the implemented method will be discussed. 


\section{Macroscopic strength criterion of a periodic composite material}

\subsection{Macroscopic strength criterion}

According to the yield design homogenization method [17-19, 24], the macroscopic criterion of a periodic composite material is obtained from solving an auxiliary yield design problem attached to the representative unit cell $\mathcal{C}$ of the composite material (see Figure 1). Denoting by $\mathbf{G}^{\text {hom }}$ the macroscopic strength domain and by $F($.$) the associated yield strength function, their definition reads$

$$
\underline{\underline{\Sigma}} \in \mathbf{G}^{h o m} \Leftrightarrow F(\underline{\underline{\Sigma}}) \leq 0 \Leftrightarrow\left\{\begin{array}{l}
\exists \underline{\underline{\sigma}} \text { statically admissible with } \underline{\underline{\Sigma}} \\
\forall \underline{\underline{\xi}} \in \mathcal{C}, f(\underline{\underline{\sigma}}(\underline{\xi})) \leq 0
\end{array}\right.
$$

where $f($.$) is the yield strength function at any point of \mathcal{C}$.

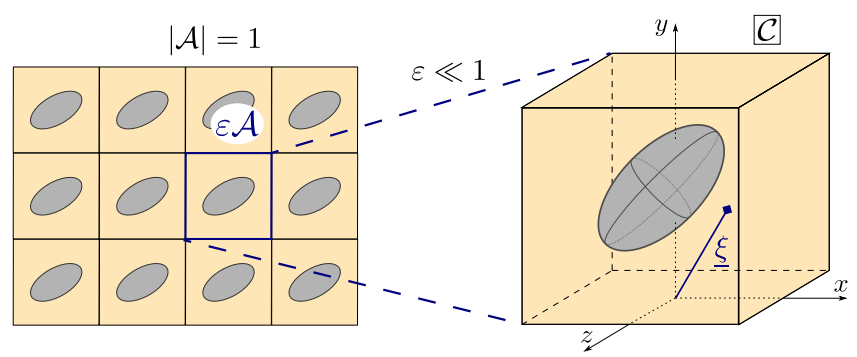

Figure 1: Representative unit cell for a periodic composite material.

A stress field $\underline{\underline{\sigma}}$ is statically admissible with $\underline{\underline{\Sigma}}$ if it complies with the following conditions:

- $\underline{\underline{\sigma}}$ is in equilibrium with no body forces:

$$
\operatorname{div} \underline{\underline{\sigma}}=0
$$

- the stress vector remains continuous across any possible discontinuity surface of the stress field:

$$
[\underline{\underline{\sigma}}] \cdot \underline{n}=0
$$

where $[\underline{\underline{\sigma}}]$ denotes the jump of $\underline{\underline{\sigma}}$ across such a surface following its unit normal $\underline{n}$.

- $\underline{\underline{\sigma}} \cdot \underline{n}$ is anti-periodic, which means that it takes opposite values at any couples of points located on opposite sides $(+$ and -$)$ of the unit cell, i.e

$$
\underline{\sigma}^{+} \cdot \underline{n}^{+}+\underline{\sigma}^{-} \cdot \underline{n}^{-}=0
$$

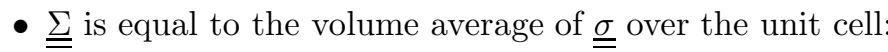

$$
\underline{\underline{\Sigma}}=\frac{1}{|\mathcal{C}|} \int_{\mathcal{C}} \underline{\underline{\sigma}} \mathrm{d} \mathcal{C}=\langle\underline{\underline{\sigma}}\rangle
$$

Equation (1) constitutes the static definition of the macroscopic strength criterion for the composite material. 


\subsection{Material strength properties}

Looking forward to applying our results to geocomposites (reinforced soils, rocks), the Mohr-Coulomb strength criterion will be adopted:

$$
f(\underline{\underline{\sigma}})=\sigma_{1}-a \sigma_{3}-k \leq 0
$$

where $\sigma_{1} \geq \sigma_{3}$ denote the two extremal eigenvalues of the stress tensor $\underline{\underline{\sigma}}$, while $a$ and $k$ may be expressed as functions of the material friction angle $\varphi$ and cohesion $C$ :

$$
\begin{gathered}
a=\frac{1-\sin \varphi}{1+\sin \varphi} \\
k=2 C \frac{\cos \varphi}{1+\sin \varphi}
\end{gathered}
$$

In order to facilitate the implementation of this criterion in a numerical method, some authors have proposed to formulate it as an optimization problem $[11,15,16,25,26]$. It can thus be proved, without any loss of generality, that the Mohr-Coulomb criterion (6) can be rewritten as two semidefinite constraints given by:

$$
\begin{gathered}
\underline{\underline{X}}^{\alpha}=-\underline{\underline{\sigma}}+(k-a \lambda) \underline{\underline{I}} \succeq 0 \\
\underline{\underline{X}}^{\beta}=\underline{\underline{\sigma}}+\lambda \underline{\underline{I}} \succeq 0
\end{gathered}
$$

where $\mathbb{I}$ is the identity tensor in three dimensions and $\lambda$ a variable only used for the coming optimization. $\underline{\underline{A}} \succeq 0$ symbolises the fact that the $n \times n$ symmetric tensor $\underline{\underline{A}}$ belongs to the positive semidefinite cone, which means that

$$
\underline{\underline{A}} \succeq 0 \Leftrightarrow \underline{x} \cdot \underline{\underline{A}} \cdot \underline{x} \geq 0, \forall \underline{x} \in \mathbb{R}^{n}
$$

Looking at (7) and (8), it is worthnoting that the above formulation remains valid for a material obeying a Tresca strength condition, which corresponds to $\varphi=0$ and then $a=1$ and $k=2 C$.

\subsection{Kinematic definition}

Thanks to the virtual work principle, a dualisation of the equilibrium equations may be performed, which allows to redefine the macroscopic strength domain as follows. For a given macroscopic strain rate tensor $\underline{\underline{D}}, \mathbf{G}^{\text {hom }}$ may be defined as:

$$
\mathbf{G}^{h o m}=\bigcap_{\underline{\underline{D}}}\left\{\underline{\underline{\underline{\Sigma}}} \mid \underline{\underline{\Sigma}}: \underline{\underline{D}} \leq \pi^{h o m}(\underline{\underline{D}})\right\}
$$

with

$$
\pi^{h o m}(\underline{\underline{D}})=\min _{\underline{U} \mathrm{ka} \underline{\underline{D}}}\left\{\langle\pi(\underline{\underline{d}})\rangle=\frac{1}{|\mathcal{C}|} \int_{\mathcal{C}} \pi(\underline{\underline{d}}) \mathrm{d} \mathcal{C}\right\}
$$

where $\underline{U}$ is a velocity field, kinematically admissible with the macroscopic strain rate tensor, that is of the form:

$$
\underline{U}(\underline{\xi})=\underline{D} \cdot \underline{\xi}+\underline{\nu}^{\text {per }}(\underline{\xi})+\text { rigid body motion }
$$


where $\underline{\nu}^{\text {per }}(\underline{\xi})$ is a periodic fluctuation.

On account of (6), (7) and (8), the support function $\pi$ of a Mohr-Coulomb material is of the form [27]:

$$
\pi(\underline{\underline{d}})= \begin{cases}C \cot \varphi \operatorname{tr} \underline{\underline{d}} & \text { if } \operatorname{tr} \underline{\underline{d}} \geq \sin \varphi\left(\left|d_{1}\right|+\left|d_{2}\right|+\left|d_{3}\right|\right) \\ +\infty & \text { otherwise }\end{cases}
$$

where $d_{1}, d_{2}$ and $d_{3}$ are the eigenvalues of the strain rate tensor $\underline{\underline{d}}$.

Using the same reasoning as for the strength condition, the equivalence of the above equation with the following system may be proved and avoids the singularity problem of (14) when $\varphi=0[16]$ :

$$
\begin{gathered}
\pi(\underline{\underline{d}})=k \operatorname{tr} \underline{\underline{d}}^{\alpha} \\
\underline{\underline{d}}=\underline{\underline{d}}^{\alpha}-\underline{\underline{d}}^{\beta} \\
-a \operatorname{tr} \underline{\underline{d}}^{\alpha}+\operatorname{tr} \underline{\underline{d}}^{\beta}=0 \\
\underline{\underline{d}}^{\alpha}, \underline{\underline{d}}^{\beta} \succeq 0
\end{gathered}
$$

where $\underline{\underline{d}}^{\alpha}$ and $\underline{\underline{d}}^{\beta}$ are two second order tensors used in the upcoming optimization.

Thus, the kinematic definition of the macroscopic strength criterion is directly expressed as a minimization of the support function for a given macroscopic strain rate. In order to obtain the most accurate lower and upper bound estimates for $\mathbf{G}^{\text {hom }}$, numerical optimization will now be performed using SDP implemented in commercial codes such as the new MOSEK software package [28].

\section{The case of unidirectionnally reinforced materials}

The rest of this paper will now investigate the yield strength behaviour of a medium reinforced by parallel fibers along one direction, indexed here by $y$. This configuration corresponds for instance to metal-matrix composites or soils reinforced by linear inclusions. This means that the composite material may be regarded as a heterogeneous medium exhibiting periodicity in the transversal directions $(x, z)$ only, the associated unit cell being displayed in Figure 2.

The stress vectors and the velocity fluctuations at two points facing each other on opposite sides, have to respect antiperiodicity and periodicity conditions respectively. These conditions are described in Figure 2, where the coordinates of the two points are $\underline{\xi}^{+}$and $\underline{\xi}^{-}$and the outer normal for each lateral side of the unit cell are denoted by $\underline{n}^{+}$and $\underline{n}^{-}$. 


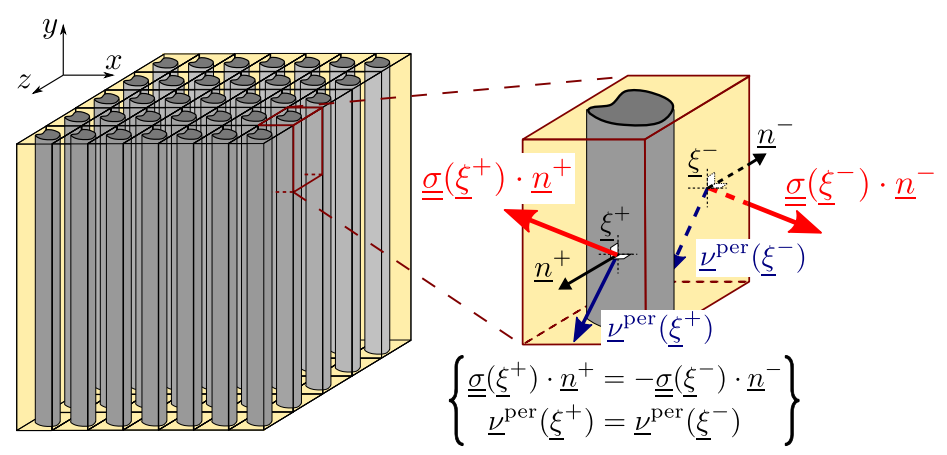

Figure 2: Representative unit cell for an unidirectional fibrous composite.

Since the material strength properties are independent of the $y$-coordinate, the stress and strain rate fields to be explored respectively in the static and kinematic approaches, are also independent of $y$ [23]. More precisely:

- the stress fields on the unit cell are of the form:

$$
\underline{\underline{\sigma}}(\underline{\xi})=\underline{\underline{\sigma}}(x, z)
$$

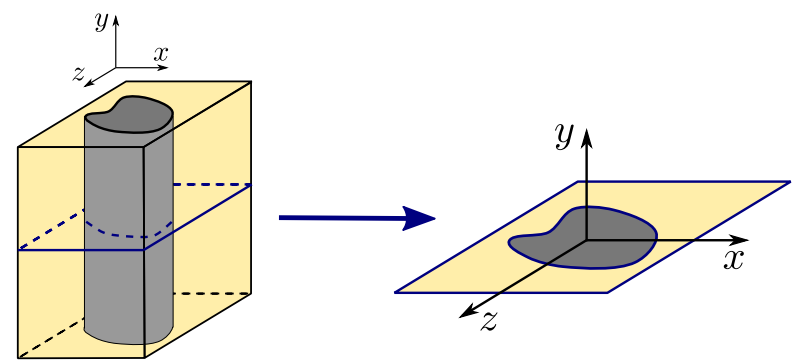

Figure 3: Representative unit cell under generalized plane strain conditions.

- whereas the velocity fields may be written as:

$$
\underline{U}(\underline{\xi})=\underline{D} \cdot \underline{\xi}+\underline{\nu}^{\mathrm{per}}(x, z) \quad \text { with } \underline{\nu}^{\mathrm{per}}(x, z)=\left(\begin{array}{c}
\tilde{u}(x, z) \\
\tilde{v}(x, z) \\
\tilde{w}(x, z)
\end{array}\right)
$$

so that the corresponding strain rate fields are:

$$
\underline{\underline{d}}(\underline{\xi})=\underline{\underline{D}}+\underline{\underline{\tilde{d}}}(x, z)
$$


with

$$
\underline{\underline{d}}(x, z)=\left(\begin{array}{ccc}
\frac{\partial \tilde{u}}{\partial x} & \frac{1}{2} \frac{\partial \tilde{v}}{\partial x} & \frac{1}{2}\left(\frac{\partial \tilde{u}}{\partial z}+\frac{\partial \tilde{w}}{\partial x}\right) \\
\frac{1}{2} \frac{\partial \tilde{v}}{\partial x} & 0 & \frac{1}{2} \frac{\partial \tilde{v}}{\partial z} \\
\frac{1}{2}\left(\frac{\partial \tilde{u}}{\partial z}+\frac{\partial \tilde{w}}{\partial x}\right) & \frac{1}{2} \frac{\partial \tilde{v}}{\partial z} & \frac{\partial \tilde{w}}{\partial z}
\end{array}\right)
$$

The initial 3D yield design auxiliary problem attached to the unit cell may therefore be reduced to that relating to the $2 \mathrm{D}$ cross section of the unit cell, as shown in Figure 3. This simplification is referred to by some authors [21, 29, 30] as generalized plane strain (GPS) conditions.

\section{Numerical implementation}

\subsection{Static approach}

Adopting a finite element formulation of the problem (1) (similar to those developed in [23]) and thanks to the GPS simplification, the unit cell cross section is discretized into $n_{e}$ three-noded triangular stress elements in the $(x, z)$ plane. Each element $e$ is associated with its own set of internal stress evaluation points (see Figure 4) and linear shape functions of $x$ and $z$ are used to interpolate the nodal stress components. For a given node $i$ in an element $e$, the components of the stress tensor $\underline{\underline{\sigma}}_{i}^{e}$ of the form

$$
\underline{\underline{\sigma}}_{i}^{e}=\left(\begin{array}{ccc}
\sigma_{x x, i}^{e} & \sigma_{x y, i}^{e} & \sigma_{x z, i}^{e} \\
\sigma_{x y, i}^{e} & \sigma_{y y, i}^{e} & \sigma_{y z, i}^{e} \\
\sigma_{x z, i}^{e} & \sigma_{y z, i}^{e} & \sigma_{z z, i}^{e}
\end{array}\right)
$$

are gathered in an array denoted by $\{\sigma\}_{i}^{e}$ and expressed as

$$
{ }^{t}\{\sigma\}_{i}^{e}=\left\{\begin{array}{llllll}
\sigma_{x x, i}^{e} & \sigma_{y y, i}^{e} & \sigma_{z z, i}^{e} & \sigma_{y z, i}^{e} & \sigma_{x z, i}^{e} & \sigma_{x y, i}^{e}
\end{array}\right\}, \quad i=1,2,3
$$

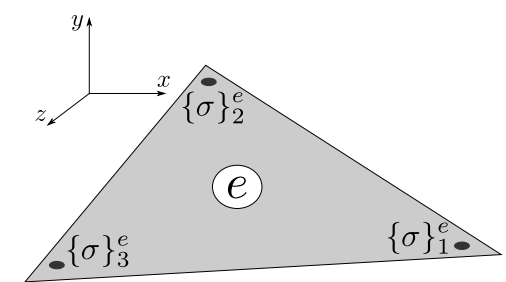

Figure 4: Internal stress evaluation points used in the static approach of yield design.

The chosen stress field has to satisfy the yield criterion in the whole composite. Due to the linear variation of the field, the respect of (9a) and (9b) on each vertex of an element $e$ ensures that the yield condition is verified at any point 
of this element. The two symmetric second order forms $\underline{\underline{X}}_{i}^{\alpha, e}$ and $\underline{\underline{X}}_{i}^{\beta, e}$ must remain positive semidefinite, and are defined at each vertex $i$ of the element by

$$
\begin{gathered}
\underline{\underline{X}}_{i}^{\alpha, e}=-\underline{\underline{\sigma}}_{i}^{e}+\left(k_{i}-a_{i} \lambda_{i}^{e}\right) \mathbb{I} \\
\underline{\underline{X}}_{i}^{\beta, e}=\underline{\underline{\sigma}}_{i}^{e}+\lambda_{i}^{e} \mathbb{I}
\end{gathered}
$$

where $a_{i}$ and $k_{i}$ are the parameters which define the strength criterion at the edge $i$ and $\lambda_{i}^{e}$ is an optimization variable.

As for $\underline{\sigma}_{i}^{e}$, these tensors are gathered in $\left\{X^{\alpha}\right\}_{i}^{e}$ and $\left\{X^{\beta}\right\}_{i}^{e}$ respectively. For each vertex, these arrays are defined by

$$
\begin{gathered}
\left\{X^{\alpha}\right\}_{i}^{e}=-\{\sigma\}_{i}^{e}-\left\{\mathbf{L}^{\alpha}\right\}_{i}^{e} \lambda_{i}^{e}+\{K\}_{i}^{e} \quad \text { with }\left\{\mathbf{L}^{\alpha}\right\}_{i}^{e}=\left\{\begin{array}{c}
a_{i} \\
a_{i} \\
a_{i} \\
0 \\
0 \\
0
\end{array}\right\} \text { and }\{K\}_{i}^{e}=\left\{\begin{array}{c}
k_{i} \\
k_{i} \\
k_{i} \\
0 \\
0 \\
0
\end{array}\right\} \\
\left\{X^{\beta}\right\}_{i}^{e}=\{\sigma\}_{i}^{e}+\left\{\mathbf{L}^{\beta}\right\}_{i}^{e} \lambda_{i}^{e} \quad \text { with }\left\{\mathbf{L}^{\beta}\right\}_{i}^{e}=\left\{\begin{array}{l}
1 \\
1 \\
1 \\
0 \\
0 \\
0
\end{array}\right\}
\end{gathered}
$$

For the sake of simplicity, the following notation is adopted:

$$
\{A\} \succeq 0 \Leftrightarrow \underline{\underline{A}} \succeq 0
$$

Thereby the positive semidefinite conditions of $\underline{\underline{X}}_{i}^{\alpha, e}$ and $\underline{\underline{X}}_{i}^{\beta, e}$ may be written as

$$
\left\{X^{\alpha}\right\}_{i}^{e},\left\{X^{\beta}\right\}_{i}^{e} \succeq 0, \quad \forall i=1,2,3 ; \forall e=1, \ldots, n_{e}
$$

It is worth noting that the respect of the yield condition introduces a new variable $\lambda_{i}^{e}$ at each vertex $i$ of each element $e$. These additional unknowns as well as $\{\sigma\}_{i}^{e}$ are gathered in two global arrays, denoted by $\{\lambda\}$ and $\{\sigma\}$ respectively, and are expressed by

$$
\{\sigma\}=\left\{\begin{array}{c}
\vdots \\
\{\sigma\}_{i}^{e} \\
\vdots
\end{array}\right\},\{\lambda\}=\left\{\begin{array}{c}
\vdots \\
\lambda_{i}^{e} \\
\vdots
\end{array}\right\} \quad i=1,2,3 ; e=1, \ldots, n_{e}
$$

Thus, there are $3 \times 7 \times n_{e}$ optimization unknowns to determine for obtaining the optimal stress field in the whole cell. Gathering all the local equations (23a) and $(23 \mathrm{~b})$, the following global equations are obtained depending on $\{\lambda\}$ and 
$\{\sigma\}$

$$
\begin{aligned}
& \{\sigma\}+\left[\mathbf{L}^{\alpha}\right]\{\lambda\}=\{K\}-\left\{X^{\alpha}\right\} \\
& \{\sigma\}+\left[\mathbf{L}^{\beta}\right]\{\lambda\}=\left\{X^{\beta}\right\}
\end{aligned}
$$

where

$$
[\bullet]=\left[\begin{array}{lll}
\ddots & & \\
& \{\bullet\}_{i}^{e} & \\
& & \ddots
\end{array}\right] \text { with } \bullet=\mathbf{L}^{\alpha}, \mathbf{L}^{\beta} \text { and }\{\cdot\}=\left\{\begin{array}{c}
\vdots \\
\{\cdot\}_{i}^{e} \\
\vdots
\end{array}\right\} \text { with } \cdot=K, X^{\alpha}, X^{\beta}
$$

Since the discretized stress field has to remain statically admissible with the macroscopic stress tensor $\underline{\underline{\Sigma}}$, Eqs. (2) to (5) must be developed for the numerical procedure. Due to the stress field invariance along the $y$-direction, the equilibrium is expressed by

$$
\underline{\operatorname{div}} \underline{\underline{\sigma}}=\underline{0} \rightarrow\left\{\begin{array}{l}
\frac{\partial \sigma_{x x}}{\partial x}+\frac{\partial \sigma_{x z}}{\partial z}=0 \\
\frac{\partial \sigma_{x y}}{\partial x}+\frac{\partial \sigma_{y z}}{\partial z}=0 \\
\frac{\partial \sigma_{x z}}{\partial x}+\frac{\partial \sigma_{z z}}{\partial z}=0
\end{array}\right.
$$

The stresses in an element $e$ are obtained from the stress arrays at each evaluation point and the shape function matrix:

$$
\{\sigma\}^{e}(x, z)=[N]^{e}(x, z)\left\{\begin{array}{l}
\{\sigma\}_{1}^{e} \\
\{\sigma\}_{2}^{e} \\
\{\sigma\}_{3}^{e}
\end{array}\right\}
$$

with

$$
[N]^{e}(x, z)=\left[N_{1}^{e}(x, z)\left[\mathbf{I}_{6}\right] \quad N_{2}^{e}(x, z)\left[\mathbf{I}_{6}\right] \quad N_{3}^{e}(x, z)\left[\mathbf{I}_{6}\right]\right]
$$

where the shape function $N_{i}^{e}(x, z)$ is defined by

$$
N_{i}^{e}(x, z)=\left\{\begin{array}{ll}
1 & \text { at the node } i \\
0 & \text { at the other nodes }
\end{array} \quad i=1,2,3\right.
$$

The discretized equilibrium could be expressed as:

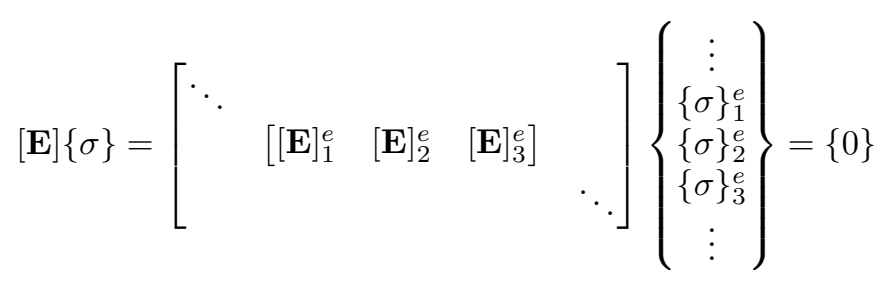


with

$$
[\mathbf{E}]_{i}^{e}=\left[\begin{array}{cccccc}
\frac{\partial N_{i}^{e}}{\partial x} & 0 & 0 & 0 & \frac{\partial N_{i}^{e}}{\partial z} & 0 \\
0 & 0 & 0 & \frac{\partial N_{i}^{e}}{\partial z} & 0 & \frac{\partial N_{i}^{e}}{\partial x} \\
0 & 0 & \frac{\partial N_{i}^{e}}{\partial z} & 0 & \frac{\partial N_{i}^{e}}{\partial x} & 0
\end{array}\right]
$$

The continuity of the stress vector accross any discontinuity surface (expressed by (3)) as well as the antiperiodicity condition (4) may be treated in the same way. For two elements $g$ and $h$ sharing a same line $l$ (or having the lines $l^{+}$and $l^{-}$facing each other), the normal $\underline{n}^{l}$ is of the form

$$
\underline{n}^{l}=\left(\begin{array}{c}
n_{x}^{l} \\
0 \\
n_{z}^{l}
\end{array}\right)
$$

The arrays of stress unknowns being denoted by $\{\sigma\}_{i}^{g},\{\sigma\}_{j}^{g}$ for the element $g$

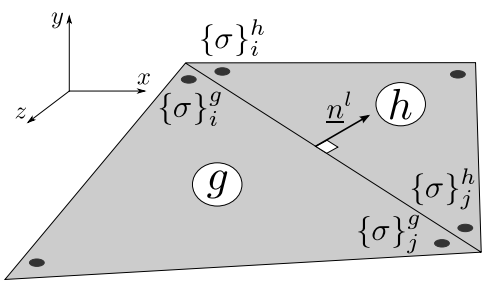

Figure 5: Two elements sharing a same line.

and $\{\sigma\}_{i}^{h},\{\sigma\}_{j}^{h}$ for the other (see Figure 5), all the above conditions may be expresed by:

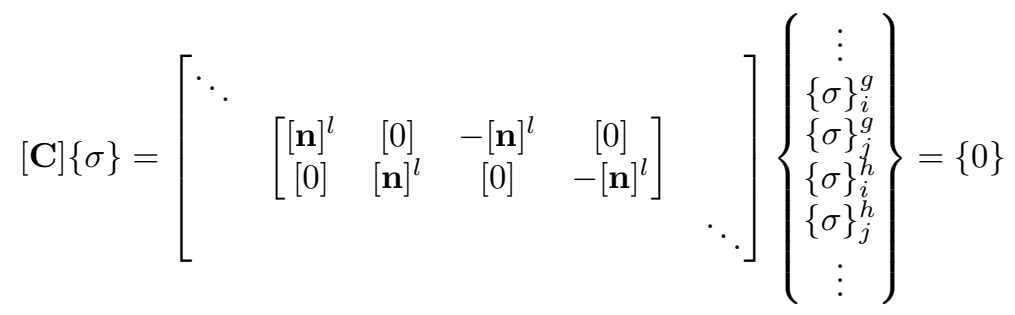

with

$$
[\mathbf{n}]^{l}=\left[\begin{array}{cccccc}
n_{x}^{l} & 0 & 0 & 0 & n_{z}^{l} & 0 \\
0 & 0 & 0 & n_{z}^{l} & 0 & n_{x}^{l} \\
0 & 0 & n_{z}^{l} & 0 & n_{x}^{l} & 0
\end{array}\right]
$$

Finally the stress field has to be statically admissible with $\underline{\underline{\Sigma}}$, that is satisfy the averaging condition (5). Thanks to the linear variation of the stress field in each element, the average of a component $\sigma_{k l}$ is calculated as:

$\Sigma_{k l}=\left\langle\sigma_{k l}\right\rangle=\frac{1}{|\mathcal{C}|} \int_{\mathcal{C}} \sigma_{k l} \mathrm{~d} \mathcal{C}=\frac{1}{|\mathcal{C}|} \sum_{e=1}^{n_{e}} \int_{e} \sigma_{k l}^{e} \mathrm{~d} \Omega_{e}=\frac{1}{|\mathcal{C}|} \sum_{e=1}^{n_{e}}\left|\Omega_{e}\right| \frac{\sigma_{k l, 1}^{e}+\sigma_{k l, 2}^{e}+\sigma_{k l, 3}^{e}}{3}$ 
where $\left|\Omega_{e}\right|$ is the area of element $e$.

A macroscopic stress tensor of the form

$$
\underline{\underline{\Sigma}}=p \underline{\underline{\Sigma}}
$$

is prescribed, where $\underline{\underline{\tilde{\Sigma}}}$ is the chosen stress direction and $p$ is the loading factor which has to be maximized in order to get the best lower bound estimate. All components of $\underline{\underline{\Sigma}}$ are gathered in the array

$$
{ }^{t}\{\tilde{\Sigma}\}=\left\{\begin{array}{llllll}
\tilde{\Sigma}_{x x} & \tilde{\Sigma}_{y y} & \tilde{\Sigma}_{z z} & \tilde{\Sigma}_{y z} & \tilde{\Sigma}_{x z} & \tilde{\Sigma}_{x y}
\end{array}\right\}
$$

and the stress loading is then imposed as follows

$$
p\{\tilde{\Sigma}\}=[\mathbf{P}]\{\sigma\}
$$

where $[\mathbf{P}]$ is defined as

$$
[\mathbf{P}]=\frac{1}{|\mathcal{C}|}\left[\begin{array}{llll}
\cdots & \frac{\left|\Omega_{e}\right|}{3}\left[\left[\mathbf{I}_{6}\right]\right. & {\left[\mathbf{I}_{6}\right]} & {\left[\mathbf{I}_{6}\right]}
\end{array}\right]
$$

Finally the optimization problem associated with the static approach of the yield design problem (1) consists in finding the maximum value of $p$ subject to the previously mentioned conditions:

$$
\max \{p \text { subject to (25), (27), (33), (36) and (41) }\}
$$

which could be expressed as:

$\max \left\{p\right.$ subject to $\left[\begin{array}{c}\left.\left[\begin{array}{cc}{\left[\mathbf{I}_{18 n_{e}}\right]} & {\left[\mathbf{L}^{\alpha}\right]} \\ {\left[\mathbf{I}_{18 n_{e}}\right]} & {\left[\mathbf{L}^{\beta}\right]} \\ {[\mathbf{E}]} & {[0]} \\ {[\mathbf{C}]} & {[0]} \\ {[\mathbf{P}]} & {[0]}\end{array}\right]\left\{\begin{array}{l}\{\sigma\} \\ \{\lambda\}\end{array}\right\}+p\left\{\begin{array}{l}\{0\} \\ \{0\} \\ \{0\} \\ \{0\} \\ -\{\tilde{\Sigma}\}\end{array}\right\}=\left\{\begin{array}{c}\{K\}-\left\{X^{\alpha}\right\} \\ \left\{X^{\beta}\right\} \\ \{0\} \\ \{0\} \\ \{0\}\end{array}\right\}\right\},\left\{X^{\beta}\right\}_{i}^{e} \succeq 0\end{array}\right\}$

where $\{\sigma\}$ and $\{\lambda\}$ are the optimization variables.

\subsection{Kinematic approach}

The implementation of the kinematic approach is based on the discretization of the unit cell cross section into $n_{e}$ six-noded triangular elements corresponding to a quadratic variation of the periodic velocity field $\underline{\nu}^{\text {per }}$, no velocity discontinuity being allowed between two adjacent elements. There are $N_{p}$ nodes in the whole cell, each with three velocity components $(\tilde{u}, \tilde{v}, \tilde{w})$. The velocity unknows for an element are then

$$
\underline{\nu}^{\text {per }} \rightarrow{ }^{t}\{\tilde{U}\}^{e}=\left\{\begin{array}{lllllll}
\tilde{u}_{1}^{e} & \tilde{v}_{1}^{e} & \tilde{w}_{1}^{e} & \cdots & \tilde{u}_{6}^{e} & \tilde{v}_{6}^{e} & \tilde{w}_{6}^{e}
\end{array}\right\}
$$


It has been shown in [10] that the kinematic inequation of (14), also expressed through Eqs.(15a) to (15d), which ensures that the corresponding support function remains finite, is satisfied at any point of the element if it is satisfied at its three vertices, provided that the sides of the element are straight segments. Each element $e$ is associated with its own set of internal strain rate evaluation points (see Figure 6). Using the same shorthand notation as in the static approach, the strain rate at vertex $i$ of the element is

$$
{ }^{t}\{d\}_{i}^{e}=\left\{d_{x x, i}^{e} \quad d_{y y, i}^{e} \quad d_{z z, i}^{e} \quad d_{y z, i}^{e} \quad d_{x z, i}^{e} \quad d_{x y, i}^{e}\right\}, \quad i=1,2,3
$$

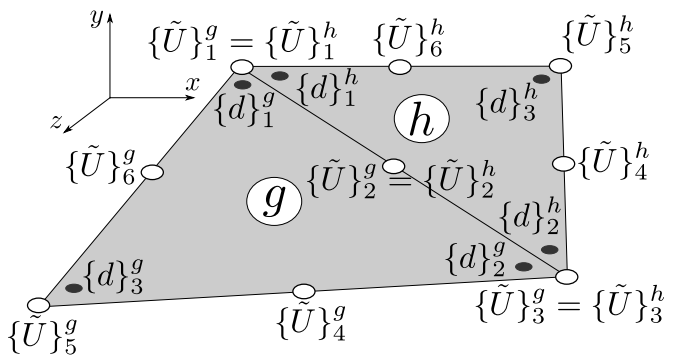

Figure 6: Internal strain rate evaluation points used for the kinematic approach.

Using Equation (18), velocity and strain rate arrays are related by

$$
\{d\}_{i}^{e}-[\mathbf{B}]_{i}^{e}\{\tilde{U}\}^{e}-\{D\}=\{0\}
$$

where the six components of the macroscopic strain rate $\underline{\underline{D}}$ are gathered in $\{D\}$, with

$$
{ }^{t}\{D\}=\left\{\begin{array}{llllll}
D_{x x} & D_{y y} & D_{z z} & D_{y z} & D_{x z} & D_{x y}
\end{array}\right\}
$$

and

$$
[\mathbf{B}]_{i}^{e}=\left[\cdots\left[\begin{array}{ccc}
\frac{\partial N_{j}^{e}}{\partial x} & 0 & 0 \\
0 & 0 & 0 \\
0 & 0 & \frac{\partial N_{j}^{e}}{\partial z} \\
0 & \frac{1}{2} \frac{\partial N_{j}^{e}}{\partial z} & 0 \\
\frac{1}{2} \frac{\partial N_{j}^{e}}{\partial z} & 0 & \frac{1}{2} \frac{\partial N_{j}^{e}}{\partial x} \\
0 & \frac{1}{2} \frac{\partial N_{j}^{e}}{\partial x} & 0
\end{array}\right] \quad \cdots\right] j=1, \ldots, 6
$$

The derivatives of the shape functions $N_{j}^{e}(x, z)$ for six-nodes triangular elements may be found for this type of element in any book dealing with finite element method. Finally the equation may be written in a global system as:

$$
\{d\}-[\mathbf{B}]\{\tilde{U}\}-[\mathbf{M}]\{D\}=\{0\}
$$

where the unknows of the problem $\{d\}$ and $\{\tilde{U}\}$ are defined as

$$
\{d\}=\left\{\begin{array}{c}
\vdots \\
\{d\}_{i}^{e} \\
\vdots
\end{array}\right\},\{\tilde{U}\}=\left\{\begin{array}{c}
\vdots \\
\{\tilde{U}\}^{e} \\
\vdots
\end{array}\right\} \quad i=1,2,3 ; e=1, \ldots, n_{e}
$$


and

$$
[\mathbf{B}]=\left[\begin{array}{lll}
\ddots & & \\
& {[\mathbf{B}]_{i}^{e}} & \\
& & \ddots
\end{array}\right],[\mathbf{M}]=\left[\begin{array}{c}
\vdots \\
{\left[\mathbf{I}_{6}\right]} \\
\vdots
\end{array}\right]
$$

So as to makes sure that the velocity field is relevant, conditions (15b) to (15d) must be respected at each vertex of each element. Thus, as for the static approach, two symmetric second order tensors $\underline{\underline{d}}^{\alpha}$ and $\underline{\underline{d}}^{\beta}$ are introduced. The corresponding arrays $\left\{d^{\alpha}\right\}_{i}^{e}$ and $\left\{d^{\beta}\right\}_{i}^{e}$ are linked with the strain rate for an element $e$ at the vertex $i$ by the equation

$$
\left\{d^{\alpha}\right\}-\left\{d^{\beta}\right\}=\left\{\begin{array}{c}
\vdots \\
\left\{d^{\alpha}\right\}_{i}^{e} \\
\vdots
\end{array}\right\}-\left\{\begin{array}{c}
\vdots \\
\left\{d^{\beta}\right\}_{i}^{e} \\
\vdots
\end{array}\right\}=\{d\}
$$

The relationship between the arrays $\left\{d^{\alpha}\right\}_{i}^{e}$ and $\left\{d^{\beta}\right\}_{i}^{e}$ is ensured by two matrix $\left[\mathbf{L}^{\alpha}\right]$ and $\left[\mathbf{L}^{\beta}\right]$, similar to those of the static approach (see Eq.(27) and Eq.(28)).

$$
-\left[\mathbf{L}^{\alpha}\right]\left\{d^{\alpha}\right\}+\left[\mathbf{L}^{\beta}\right]\left\{d^{\beta}\right\}=\{0\}
$$

Moreover, both tensors must be positive semidefinite:

$$
\left\{d^{\alpha}\right\}_{i}^{e},\left\{d^{\beta}\right\}_{i}^{e} \succeq 0, \quad \forall i=1,2,3 ; \forall e=1, \ldots, n_{e}
$$

Finally, in order to satisfy the periodicity of the velocity field, we need to impose a relationship between nodes on two lines $\left(l^{+}\right.$and $\left.l^{-}\right)$belonging to the lateral boundary of the cell and facing each other. This may be simply expressed by:

$$
\{\tilde{U}\}_{i^{+}}-\{\tilde{U}\}_{i^{-}}=\{0\} \quad i=1,2,3
$$

with

$$
{ }^{t}\{\tilde{U}\}_{i^{ \pm}}=\left\{\tilde{u}_{i^{ \pm}}^{e} \quad \tilde{v}_{i^{ \pm}}^{e} \quad \tilde{w}_{i^{ \pm}}^{e}\right\}
$$

where the node $i^{+}$belongs to the line $l^{+}$, facing the node $i^{-}$on the line $l^{-}$.

The set of all periodicity condtions can be written as:

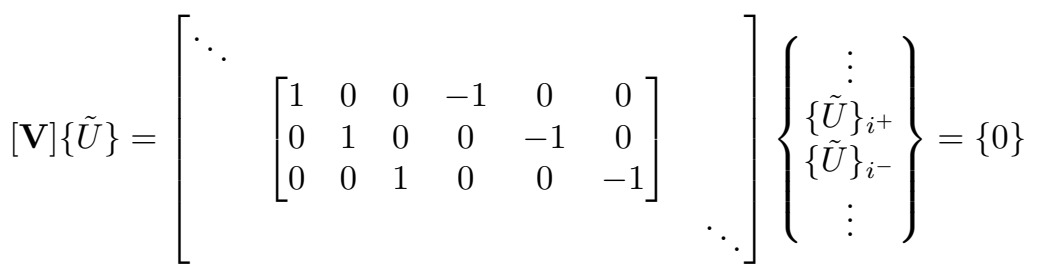

The average of the support function on the unit cell (Eq.(12)) may be expressed as a linear function of the vector $\left\{d^{\alpha}\right\}$ :

$\langle\pi(\underline{\underline{d}})\rangle=\frac{1}{|\mathcal{C}|} \sum_{e=1}^{n_{e}} \int_{e} \pi^{e}(\underline{\underline{d}}) \mathrm{d} \Omega_{e}=\frac{1}{|\mathcal{C}|} \sum_{e=1}^{n_{e}}\left|\Omega_{e}\right| \frac{\pi^{e}\left(\underline{\underline{d}}_{1}^{e}\right)+\pi^{e}\left(\underline{\underline{d}}_{2}^{e}\right)+\pi^{e}\left(\underline{\underline{d}}_{3}^{e}\right)}{3}=\frac{{ }^{t}\{\Pi\}\left\{d^{\alpha}\right\}}{|\mathcal{C}|}$ 
where $\left|\Omega_{e}\right|$ is the area of element $e$ and $\{\Pi\}$ is given by

$$
{ }^{t}\{\Pi\}=\left\{\cdots \quad\left\{\begin{array}{lllllll}
1 & 1 & 1 & 0 & 0 & 0 & 0 \\
k_{i} \frac{\left|\Omega_{e}\right|}{3} & \cdots
\end{array}\right\}\right.
$$

The best upper bound estimate $\mathbf{G}^{\mathrm{ub}}$ of the macroscopic strength domain is defined by its support function $\pi^{\mathrm{ub}}$, solution of the following optimization problem

$$
\min \{\langle\pi(\underline{\underline{d}})\rangle \text { subject to (50), (53), (54), (55) and (58) }\}
$$

Taking the discretized forms of the objective function and the constraints into account, the minimization problem is replaced by:

$$
\pi^{\mathrm{ub}}=\min \left\{\begin{array}{c}
\left\{d^{\alpha}\right\}_{i}^{e},\left\{d^{\beta}\right\}_{i}^{e} \succeq 0 \\
\frac{{ }\{\Pi\}\left\{d^{\alpha}\right\}}{|\mathcal{C}|} \text { subject to }
\end{array}\left[\begin{array}{c}
-\left[\mathbf{L}^{\alpha}\right]\left\{d^{\alpha}\right\}+\left[\mathbf{L}^{\beta}\right]\left\{d^{\beta}\right\}=\{0\} \\
{\left[\begin{array}{cc}
{\left[\mathbf{I}_{18 n_{e}}\right]} & -[\mathbf{B}] \\
{\left[\mathbf{I}_{18 n_{e}}\right]} & {[0]} \\
{[0]} & {[\mathbf{V}]}
\end{array}\right]\left\{\begin{array}{c}
\{d\} \\
\{\tilde{U}\}
\end{array}\right\}=\left\{\begin{array}{c}
{[\mathbf{M}]\{D\}} \\
\left\{d^{\alpha}\right\}-\left\{d^{\beta}\right\} \\
\{\tilde{0}\}
\end{array}\right\}}
\end{array}\right\}\right.
$$

which resolution is performed using MOSEK software package.

\section{Application to a stone column reinforced soil}

\subsection{Problem statement and boundary conditions}

Since decades, improvement techniques have been increasingly adopted in order to solve the problem due to the poor performance of soils. One of them consists in incorporating into a purely cohesive soil (seen as a matrix), a periodic distribution of reinforcing inclusions made of either cohesive and/or highly frictional material. A classical technique is the stone column reinforcement, where some ballast is introduced into the soil mass with a regular pattern. Such a reinforced soil can be viewed as two-materials periodic composite.

For this kind of reinforcement, the unit cell is a square shape cylinder of side $L$, which represents the distance between two neighboring columns, with a column placed at its center (see Figure 7 ). The radius of the column being denoted by $\rho$, the volume fraction of the reinforced soil, defined as the ratio between the volume of the domain $\mathcal{C}_{r}$ occupied by the reinforcing column and the volume of the whole unit cell, is therefore equal to

$$
\eta=\frac{\pi \rho^{2}}{L^{2}}
$$

The native soil is considered as a purely cohesive material obeying a Tresca strength criterion (cohesion denoted by $C_{m}$ ), while the reinforcing column material is a cohesive-frictional material obeying a Mohr-Coulomb condition with 


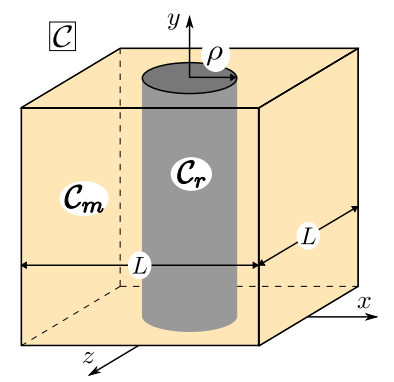

Figure 7: Representative volume element for a stone column reinforced soil.

a cohesion $C_{r}$ and a friction angle $\varphi_{r}$. The corresponding strength conditions are:

$$
\begin{array}{ll}
f^{m}(\underline{\underline{\sigma}})=\sigma_{1}-\sigma_{3}-2 C_{m} \leq 0 & \text { for the soil } \\
f^{r}(\underline{\underline{\sigma}})=\sigma_{1}-\frac{1-\sin \varphi_{r}}{1+\sin \varphi_{r}} \sigma_{3}-2 C_{r} \frac{\cos \varphi_{r}}{1+\sin \varphi_{r}} \leq 0 & \text { for the reinforcement }
\end{array}
$$

The analysis will be focused on determining the reinforced soil's macroscopic strength domain $\mathbf{G}_{c o l}^{\text {hom }}$ subject to plane strain conditions in the $(x, y)$-plane that is prescribing a macroscopic strain rate tensor of the form:

$$
\underline{\underline{D}}(\gamma, \delta)=\left(\begin{array}{ccc}
\cos \gamma \cos \delta & \frac{1}{2} \sin \delta & 0 \\
\frac{1}{2} \sin \delta & \sin \gamma \cos \delta & 0 \\
0 & 0 & 0
\end{array}\right)
$$

where the angles $\gamma$ and $\delta$ specify the orientation of $\underline{\underline{D}}$ in the space $\left(D_{x x}, D_{y y}, 2 D_{x y}\right)$, as shown in Figure 8(b).

Owing to the symmetry of the unit cell with respect to the $O x y$-plane, in terms of geometry, loading conditions and material strength properties, combined with periodicity boundary conditions enforced on the sides of the square unit cell parallel to the $x$-axis, it can be proved [31] that only one half of the unit cell need to be considered with the following boundary conditions (Figure $8(\mathrm{a}))$ :

$$
\sigma_{x z}=\sigma_{y z}=0 \text { and } U_{z}=0 \quad \text { for } z=0 \text { or }-L / 2
$$

which means that both sides are in smooth contact with fixed planes. Such boundary conditions are completed by periodicity conditions on the two remaining sides of the cell parallel to the $z$-axis, while velocities are imposed to be null on two points of the half-cell, thus removing any global rigid body motion.

Similarly, as regards the implementation of the lower bound static approach, 


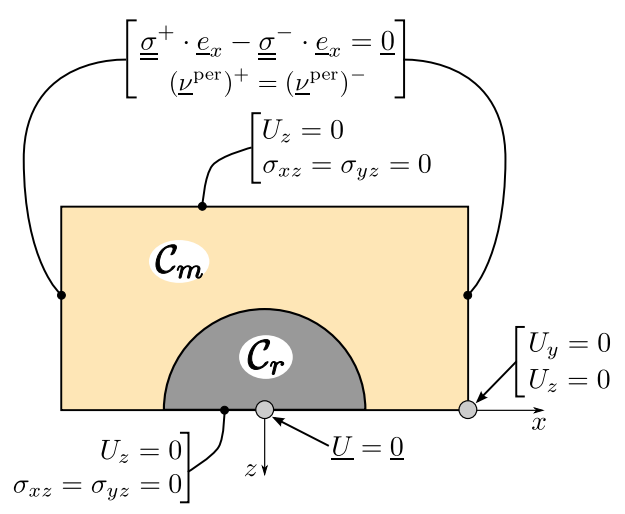

(a)

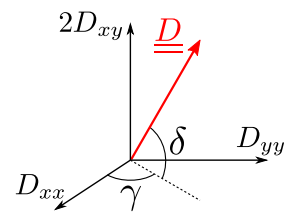

(b)

Figure 8: Boundary conditions for the determination of the plane strain macroscopic strength criterion (a) and macroscopic strain rate directions (b).

the macroscopic stress tensor is of the form:

$$
\underline{\underline{\Sigma}}(\gamma, \delta)=\left(\begin{array}{ccc}
\cos \gamma \cos \delta & \sin \delta & 0 \\
\sin \delta & \sin \gamma \cos \delta & 0 \\
0 & 0 & \Sigma_{z z}
\end{array}\right)
$$

where the out-of-plane stress component $\Sigma_{z z}$ may take any value.

5.2. Lower and upper bound estimates for the macroscopic strength criterion

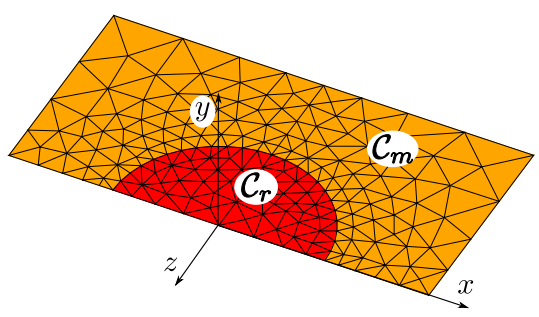

Figure 9: Finite element model adopted for obtaining the numerical lower and upper bounds.

An illustrative example will now be considered corresponding to the following values:

$$
\eta=0.2, \quad C_{r}=2 C_{m}, \quad \varphi_{r}=25^{\circ}
$$

The mesh adopted for the static and kinematic approaches will be the same, represented in Figure $9\left(n_{e}=340\right.$ elements, $N_{p}=715$ nodes for the kinematic approach).

For both approaches angles $\gamma$ and $\delta$, previously introduced, take discrete values with an increment of one degree. The optimization problems (44) and 
(62) are performed on a DELL PC (Intel-P4 2.4GHz) running Linux 32-Bits using the release 7 of MOSEK in the Matlab (2010a) environment. This recently developed version allows to treat problems using SDP. In this SDP optimizer, the global arrays which refer to the tensors respecting a semi-definite cone (in our case $\left\{X^{\alpha}\right\},\left\{X^{\beta}\right\}$ for the static approach and $\left\{d^{\alpha}\right\},\left\{d^{\beta}\right\}$ for the kinematic approach) are given as additional unknowns (see [28] for more details).

Figure 10 depicts the strength domain corresponding to the upper bound of $\mathbf{G}_{\text {col }}^{\text {hom }}$ in the space of non dimensional stresses $\left(\Sigma_{x x} / C_{m}, \Sigma_{y y} / C_{m}, \Sigma_{x y} / C_{m}\right)$. As expected when looking at the yield criterion of each constituent of this composite, there are many directions which don't admit any limit load, so that the support function value becomes infinite.

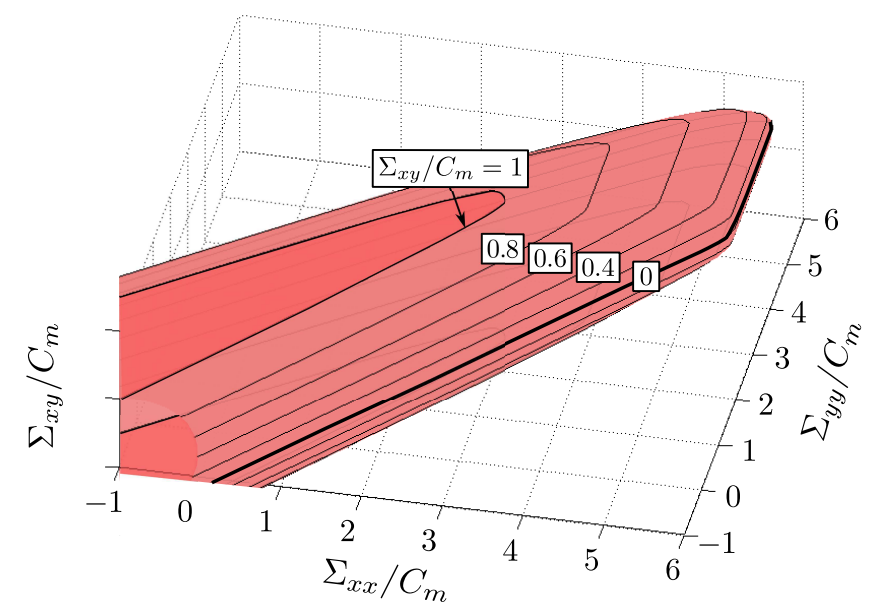

Figure 10: Upper bound of the macroscopic strength domain of a stone column reinforced soil.

More precisely, the set of macroscopic strains directions generating a finite limit load is a cone which is represented in Figure 11(a) in the $(\gamma, \delta)$-plane. It is worth noting in particular that for a pure shear stress sollicitation, the strength of the reinforced soil cannot exceed the cohesion of the native soil $C_{m}$. This remark holds true whatever the value taken by the cohesion of the reinforcement $C_{r}$.

The cross sections of this yield surface planes of constant shear stress values $\left(\Sigma_{x y}=\right.$ cst.) are displayed in Figure 11 as well as those corresponding to the lower bound $\mathbf{G}_{c o l}^{\mathrm{lb}}$. Moreover for $\Sigma_{x y} / C_{m}=0$, the soil and reinforcement individual criteria are sketched. The improvement of the native soil is clearly highlighted here for high compressive stress states, due to the reinforcing material. On the other hand, in the region of tensile stresses, the strength of the native soil is reduced since the column is a frictional material. 
From a numerical point of view, it is interesting to analyse the relative gap between the so-obtained lower and upper bounds (see Fig.11(a)). It varies between $0.3 \%$ and $3.4 \%$, with an average value calculated over all the orientations equal to $1.8 \%$.

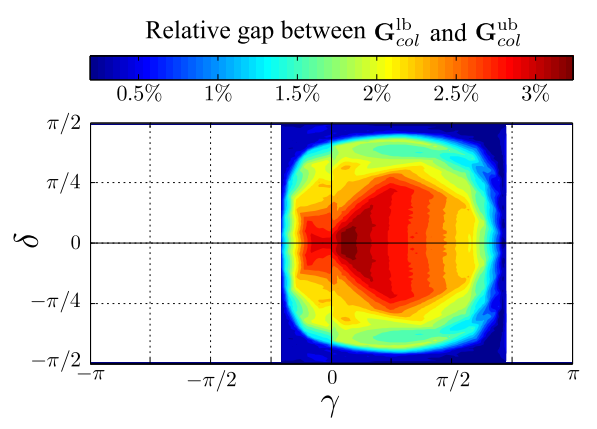

(a)

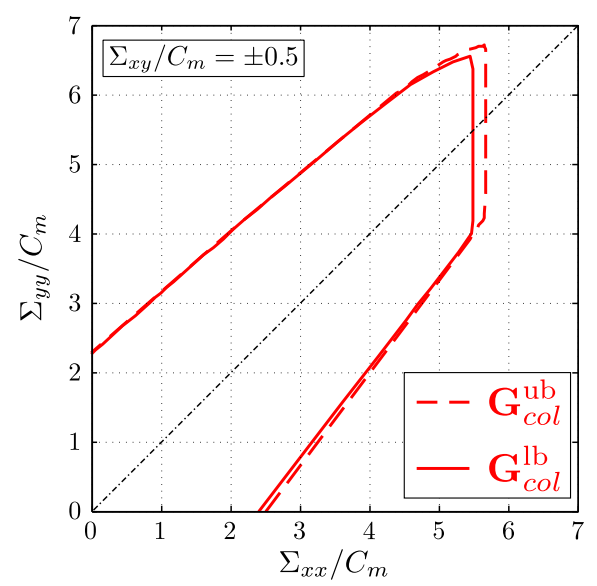

(c)

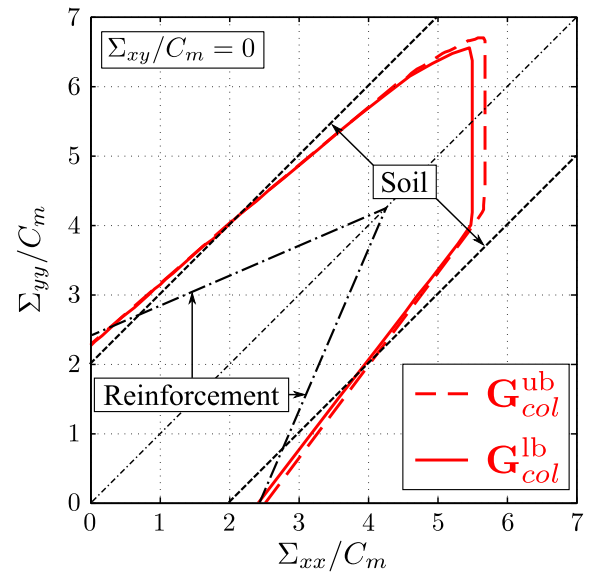

(b)

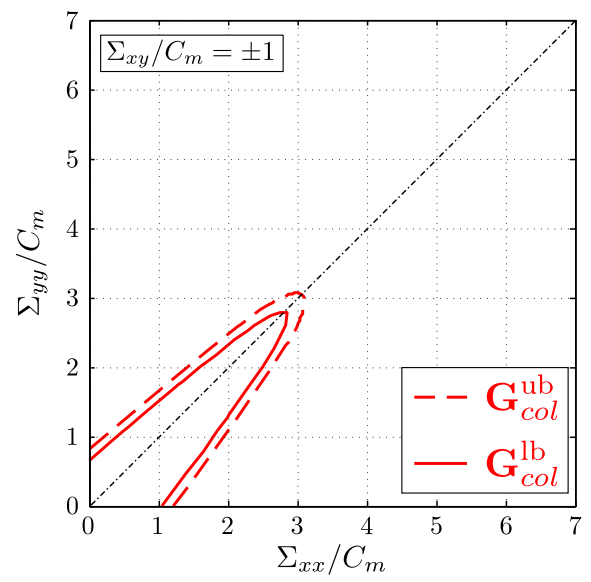

(d)

Figure 11: Cross sectioned views of the macroscopic strength criterion of a stone column reinforced soil.

This relative gap is calculated using the support functions of $\mathbf{G}_{c o l}^{\mathrm{lb}}$ and $\mathbf{G}_{c o l}^{\mathrm{ub}}$. These support functions may be expressed by:

$$
\pi^{\text {num }}=\sup _{\underline{\underline{\Sigma}}}\left\{\underline{\underline{\Sigma}}: \underline{\underline{D}}(\gamma, \delta) \mid \underline{\underline{\Sigma}} \in \mathbf{G}^{\text {num }}\right\}
$$

Table 1 gives the values taken by these support functions for a specific macro- 
scopic strain rate orientation $D_{y y}=0$ (which corresponds to $\gamma=0$ ).

The computational times required for creating the global arrays associated to (44) or (62) on the one hand and for performing one above numerical optimization on the other hand, are given in Table 2 for the determination of the entire lower bound surface $\mathbf{G}_{c o l}^{\mathrm{lb}}$ as well as the upper bound surface $\mathbf{G}_{c o l}^{\mathrm{ub}}$.

\begin{tabular}{ccccc|ccccc}
\hline$\delta$ & $\pi_{c o l}^{\mathrm{lb}}$ & $\pi_{c o l}^{\mathrm{ub}}$ & $\pi_{c t}^{\mathrm{lb}}$ & $\pi_{c t}^{\mathrm{ub}}$ & $\delta$ & $\pi_{c o l}^{\mathrm{lb}}$ & $\pi_{c o l}^{\mathrm{ub}}$ & $\pi_{c t}^{\mathrm{bl}}$ & $\pi_{c t}^{\mathrm{ub}}$ \\
\hline 0 & 5.526 & 5.695 & 4.289 & 4.299 & $5 \pi / 18$ & 3.726 & 3.805 & 2.757 & 2.761 \\
$\pi / 18$ & 5.457 & 5.614 & 4.224 & 4.234 & $\pi / 3$ & 3.001 & 3.056 & 2.145 & 2.146 \\
$\pi / 9$ & 5.231 & 5.370 & 4.030 & 4.041 & $7 \pi / 18$ & 2.205 & 2.237 & 1.699 & 1.702 \\
$\pi / 6$ & 4.862 & 4.985 & 3.714 & 3.718 & $4 \pi / 9$ & 1.519 & 1.527 & 1.438 & 1.441 \\
$2 \pi / 9$ & 4.354 & 4.452 & 3.286 & 3.288 & $\pi / 2$ & 1.000 & 1.000 & $+\infty$ & $+\infty$ \\
\hline
\end{tabular}

Table 1: Support functions of the lower and upper bound surfaces for $D_{y y}=0$ (i.e $\gamma=0$ ).

\begin{tabular}{cccccc}
\hline $\begin{array}{c}\text { Macroscopic } \\
\text { domain }\end{array}$ & $\begin{array}{c}\text { Numbers of } \\
\text { elements } n_{e}\end{array}$ & $\begin{array}{c}\text { Numbers of } \\
\text { nodes } N_{p}\end{array}$ & $\begin{array}{c}\text { Numbers of } \\
\text { unknowns }\end{array}$ & $\begin{array}{c}\text { CPU time for } \\
\text { arrays construction }\end{array}$ & $\begin{array}{c}\text { CPU time for } \\
\text { each optimization }\end{array}$ \\
\hline $\mathbf{G}_{c o l}^{\mathrm{lb}}$ & 340 & 188 & 19381 & $3.39 \mathrm{~s}$ & $3.64 \mathrm{~s}$ \\
$\mathbf{G}_{c o}^{\mathrm{ub}}$ & 340 & 715 & 20505 & $4.30 \mathrm{~s}$ & $5.51 \mathrm{~s}$ \\
$\mathbf{G}_{c t}^{\mathrm{ub}}$ & 304 & 178 & 17329 & $2.91 \mathrm{~s}$ & $2.61 \mathrm{~s}$ \\
$\mathbf{G}_{c t}^{\mathrm{ub}}$ & 304 & 659 & 18393 & $3.42 \mathrm{~s}$ & $4.36 \mathrm{~s}$ \\
\hline
\end{tabular}

Table 2: Computation times for the determination of the lower and upper bound surfaces.

Such calculation times may be compared with those resulting from the use of an elastoplastic numerical procedure. Indeed, the latter procedure, often used in order to obtain the strength domain of a material, is implemented in several finite element codes. Even though it allows to have more information than the method presented here (namely along the stress path during the loading increase), this procedure may quickly become slow and tedious, since many increments are necessary to capture the limit load. Authors have made such calculations with a similar computer and commercial finite element code, with von Mises and Drucker-Prager materials, which have taken several days with the same discretization of the stress space [22].

With a dedicated homemade finite element code which permits to treat Tresca and Mohr-Coulomb materials in an elastoplastic procedure, the so-obtained macroscopic strength domain is in quite good agreement with the present results. Nevertheless, the computation time for one macroscopic strain rate direction is evaluated to 105 seconds, whereas it takes less than 10 seconds with the numerical approaches presented here. Moreover, there is no guarantee that the solution obtained with the elastoplastic procedure has a specific (lower or upper bound) status compared to the exact strength criterion. On the contrary, the approaches developed here ensure that $\mathbf{G}_{c o l}^{\text {hom }}$ is framed by these two bounds estimates. 


\section{Macroscopic strength criterion of a cross trench reinforced soil}

A recently developed reinforcement technique is the so-called cross trench configuration. The frictional reinforcing material is introduced in the native soil in the form of a network of two perpendicular arrays of trenches, in much the same way as a "honeycomb structure". With $L$ representing the distance between two neighboring parallel arrays and $t$ denoting the thickness of the orthogonal trenches (see Figure 12), the volume fraction is defined as

$$
\eta=\frac{t(2 L-t)}{L^{2}}
$$

Classically, the thickness of the so-constructed trenches vary between $30 \mathrm{~cm}$ to $50 \mathrm{~cm}, L$ may be equal to $4 \mathrm{~m}$ to $6 \mathrm{~m}$ and the depth of one trench may reach $40 \mathrm{~m}$. These dimensional values permit to use the generalized plane strain assumptions.

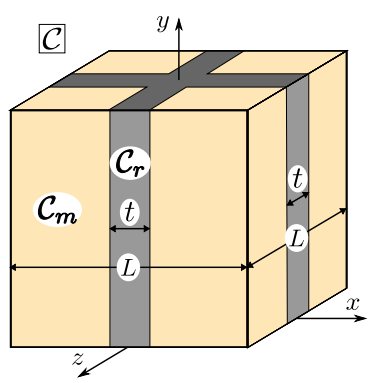

(a)

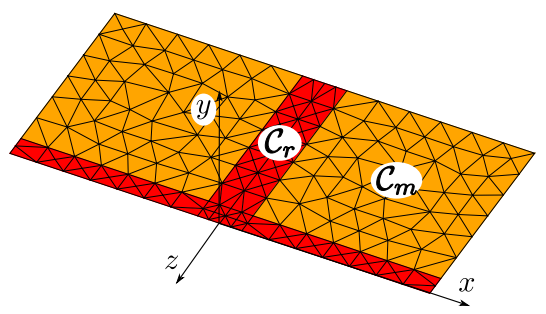

(b)

Figure 12: representative volume element and finite element mesh for a cross trench reinforced soil.

The soil and the reinforcing material are supposed to obey the yield criteria defined with the same values as those given in (68). A quite similar method is used to determine the lower and the upper bounds estimates for such a reinforced soil. The mesh chosen to do so is sketched in Figure 12. The characteristic values of this mesh are $n_{e}=304$ elements and $N_{p}=659$ nodes. For this specific geometry of reinforced soil, it appears that the fineness of the adopted mesh has a very small influence on the obtained bounds.

The computation times needed to obtain $\mathbf{G}_{c t}^{\mathrm{lb}}$ and $\mathbf{G}_{c t}^{\mathrm{ub}}$ are given in Table 2, using the same computer than for the column reinforced soil. The relative gap between the two bounds vary between 0 and $1.2 \%$ and the average on all the strain rate directions generating a limit load is equal to $0.4 \%$ (see Fig.14(a)). These results, as well as the ones given in Table 1, do qualify the method developed in the previous sections. 


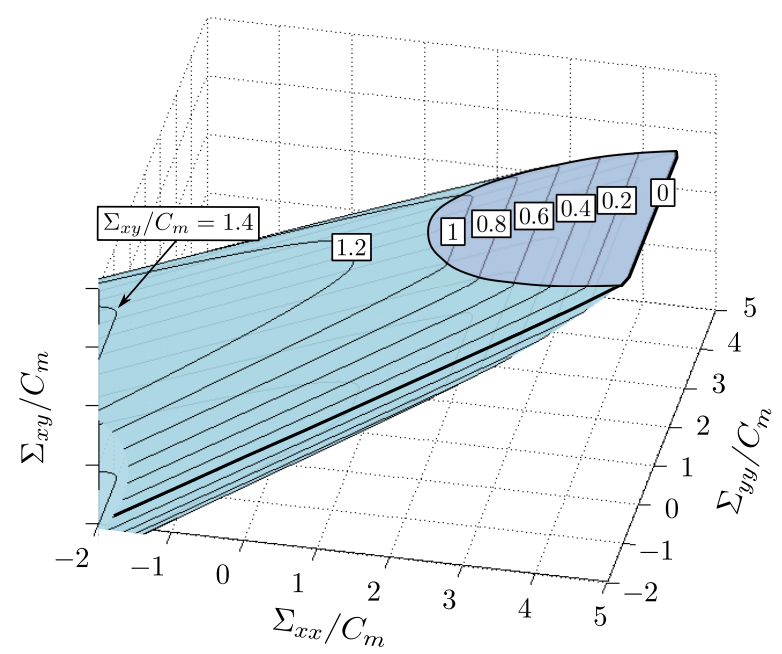

Figure 13: Upper bound of the macroscopic strength domain of a cross trench reinforced soil.

As for the stone column reinforced soil, the upper bound estimate of $\mathbf{G}_{c t}^{\text {hom }}$ is represented in the space $\left(\Sigma_{x x} / C_{m}, \Sigma_{y y} / C_{m}, \Sigma_{x y} / C_{m}\right)$. Contrary to the stone column configuration, the strength of the reinforced soil under a pure shear sollicitation is no longer limited. The set of macroscopic strain rate directions having a limit load, which can be seen as the cone of the outer normals to the macroscopic strength domain, is still a cone. Unlike for the stone column reinforced soil, the pure shear strain rate direction does not belong to this cone, which is similar to the case of the classical Mohr-Coulomb criterion (see Fig.14(a)).

The cross sections of the yield surfaces of the bounds estimates are displayed in Figure 14 with different fixed values for $\Sigma_{x y} / C_{m}$. It confirms that the soil reinforcement methods with highly frictional materials are efficient for high compressive stresses, while native soil strength properties are weakened for tensile stress states.

\section{Concluding remarks}

In this paper, a quite recently developed yield design numerical method, has been applied to the determination of the macroscopic strength properties of column or cross trench reinforced soils. It is based upon the semidefinite programming technique which makes it possible to handle 3D yield design optimization problems involving a Mohr-Coulomb strength condition. Simplifications attached to the generalized plane strain conditions have been made, leading to a significant reduction of the unknowns of the optimization problem and then of the computational times needed for performing the calculation. 


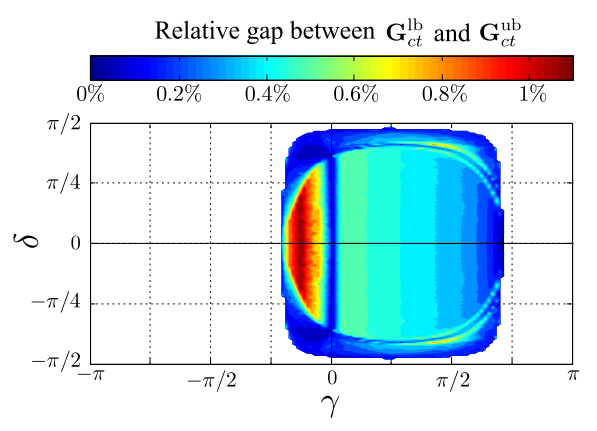

(a)

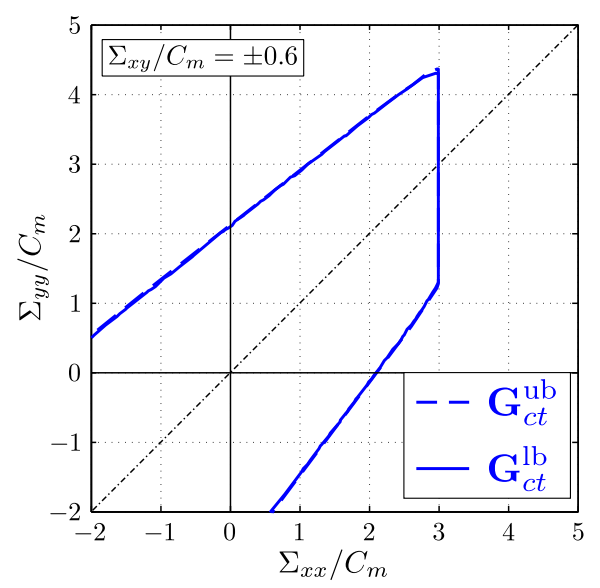

(c)

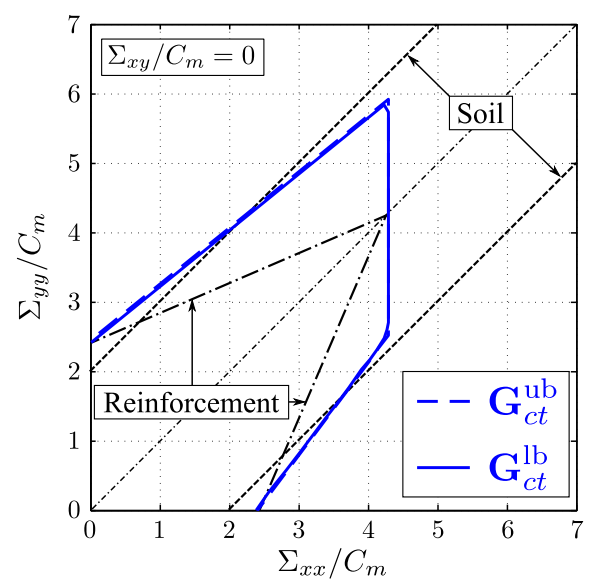

(b)

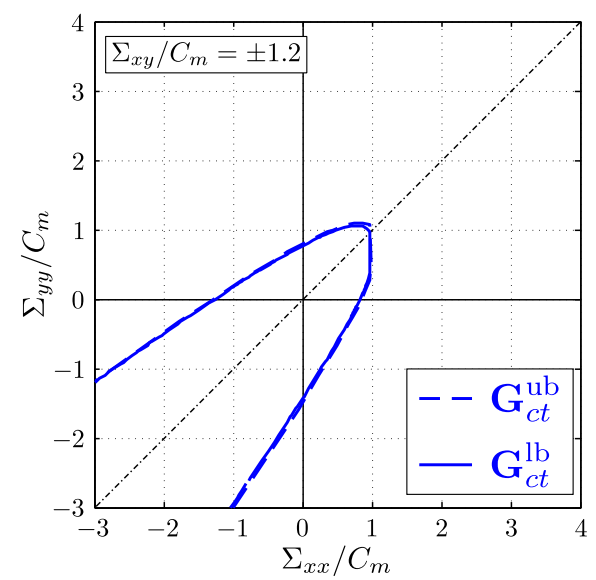

(d)

Figure 14: Bounds of the macroscopic strength criterion of a cross trench reinforced soil with $\Sigma_{x y} / C_{m}$ fixed. 


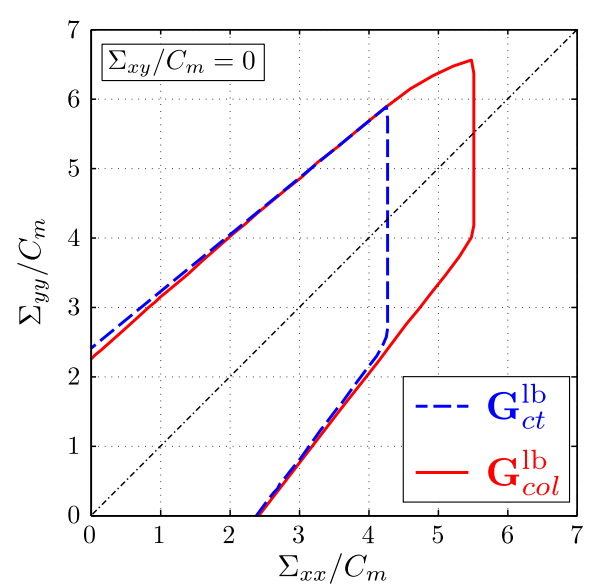

(a)

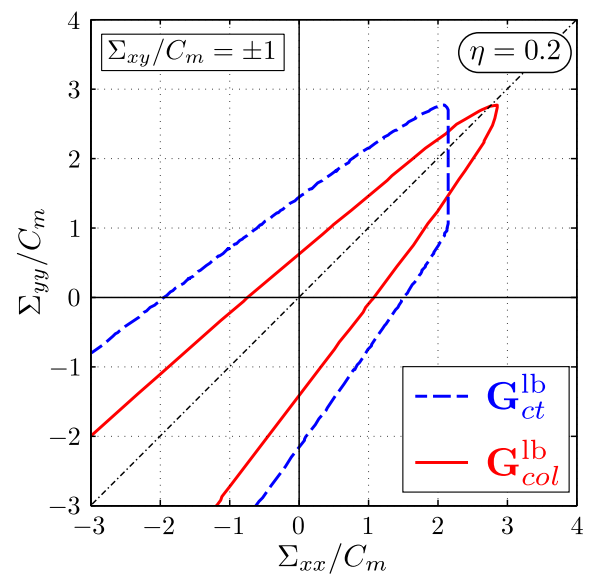

(b)

Figure 15: Comparison of the two reinforced soils configurations.

The use of numerical yield design or limit analysis represents a much more efficient alternative to elastoplastic procedures, as far as the objective of the study is to derive limit loads. Based on two illustrative examples in the area of geotechnical engineering, the numerical performance of the method has been proven, reducing the computational time from days to minutes. The accuracy can be also noticed in terms of gap between the so-obtained lower and upper bounds, which doesn't exceed a few percents in these examples.

Owing to the homogenization method, the macroscopic strength domains of a stone column and a cross trench reinforced soils have been framed by close bounds. It appears that the criterion of these composite materials is highly anisotropic due to the orientation of reinforcements. Figure 15 displays a comparison between the (lower bound) estimates to the macroscopic strength criterion of a stone column and cross trench reinforced soil, with the same value of reinforcement volume fraction. It indicates that the column reinforced soil exhibits higher strength properties than the cross trench reinforced soil, for solicitations applied transversaly to the reinforcement direction, whereas the latter reinforcing technique appears to be more efficient in the region of compressive stresses, notably for the significant values of the shear stress (Fig.15(b)).

In a forthcoming paper, the numerical optimization technique presented hereby, will be applied to the evaluation of the load bearing capacities of reinforced soil structures, making use of the macroscopic strength estimates of reinforced soils obtained in this paper. 


\section{References}

[1] J. Lysmer, Limit analysis of plane problems in soil mechanics, Journal of the Soil Mechanics and Foundations 96 (1970) 1311-1334.

[2] J. Pastor, S. Turgeman, Mise en œuvre numérique des méthodes de l'analyse limite pour les matériaux de Von Mises et de Coulomb standards en déformation plane, Mechanics Research Communications 3 (6) (1976) 469-474.

[3] S. W. Sloan, Lower bound limit analysis using finite elements and linear programming, International Journal for Numerical and Analytical Methods in Geomechanics 12 (1) (1988) 61-77.

[4] R. Abdi, P. de Buhan, J. Pastor, Calculation of the critical height of a homogenized reinforced soil wall: a numerical approach, International Journal for Numerical and Analytical Methods in Geomechanics 18 (7) (1994) 485505 .

[5] N. Zouain, J. Herskovits, L. A. Borges, R. A. Feijóo, An iterative algorithm for limit analysis with nonlinear yield functions, International Journal of Solids and Structures 30 (10) (1993) 1397-1417.

[6] A. V. Lyamin, S. W. Sloan, Lower bound limit analysis using non-linear programming, International Journal for Numerical Methods in Engineering 55 (5) (2002) 573-611.

[7] K. Krabbenhøft, L. Damkilde, A general non-linear optimization algorithm for lower bound limit analysis, International Journal for Numerical Methods in Engineering 56 (2) (2003) 165-184.

[8] M. Trillat, J. Pastor, Limit analysis and Gurson's model, European Journal of Mechanics-A/Solids 24 (5) (2005) 800-819.

[9] A. Makrodimopoulos, C. M. Martin, Lower bound limit analysis of cohesive-frictional materials using second-order cone programming, International Journal for Numerical Methods in Engineering 66 (4) (2006) 604634.

[10] A. Makrodimopoulos, C. M. Martin, Upper bound limit analysis using simplex strain elements and second-order cone programming, International Journal for Numerical and Analytical Methods in Geomechanics 31 (6) (2007) 835-865.

[11] K. Krabbenhøft, A. V. Lyamin, S. W. Sloan, Formulation and solution of some plasticity problems as conic programs, International Journal of Solids and Structures 44 (5) (2007) 1533-1549.

[12] F. Pastor, D. Kondo, J. Pastor, Numerical limit analysis bounds for ductile porous media with oblate voids, Mechanics Research Communications 38 (5) (2011) 350-354. 
[13] G. Garcea, L. Leonetti, A unified mathematical programming formulation of strain driven and interior point algorithms for shakedown and limit analysis, International Journal for Numerical Methods in Engineering 88 (11) (2011) 1085-1111.

[14] J. J. Muñoz, A. Huerta, J. Bonet, J. Peraire, A note on upper bound formulations in limit analysis, International Journal for Numerical Methods in Engineering 91 (8) (2012) 896-908.

[15] K. Krabbenhøft, A. V. Lyamin, S. W. Sloan, Three-dimensional MohrCoulomb limit analysis using semidefinite programming, Communications in Numerical Methods in Engineering 24 (11) (2008) 1107-1119.

[16] C. M. Martin, A. Makrodimopoulos, Finite-Element Limit Analysis of Mohr-Coulomb Materials in 3D Using Semidefinite Programming, Journal of Engineering Mechanics 134 (4) (2008) 339-347.

[17] P. Suquet, Elements of homogenization for inelastic solid mechanics, in: Homogenization Techniques for Composite Media, 278, 193-278, 1987.

[18] P. de Buhan, A fundamental approach to the yield design of reinforced soil structures (in French), Thèse d'Etat, paris VI, 1986.

[19] B. Jellali, M. Bouassida, P. de Buhan, A homogenization method for estimating the bearing capacity of soils reinforced by columns, International Journal for Numerical and Analytical Methods in Geomechanics 29 (10) (2005) 989-1004.

[20] M. Abdelkrim, P. de Buhan, An elastoplastic homogenization procedure for predicting the settlement of a foundation on a soil reinforced by columns, European Journal of Mechanics-A/Solids 26 (4) (2007) 736-757.

[21] A. Taliercio, Generalized plane strain finite element model for the analysis of elastoplastic composites, International Journal of Solids and Structures 42 (8) (2005) 2361-2379.

[22] G. Hassen, M. Gueguin, P. de Buhan, A homogenization approach for assessing the yield strength properties of stone column reinforced soils, European Journal of Mechanics-A/Solids 37 (2013) 266-280, dOI:10.1016/j.euromechsol.2012.07.003.

[23] P. Francescato, J. Pastor, Lower and upper numerical bounds to the offaxis strength of unidirectional fiber-reinforced composites by limit analysis methods, European Journal of Mechanics-A/Solids 16 (2) (1997) 213-234.

[24] P. de Buhan, A. Taliercio, A homogenization approach to the yield strength of composite materials, European Journal of Mechanics-A/Solids 10 (2) (1991) 129-154. 
[25] C. D. Bisbos, P. M. Pardalos, Second-order cone and semidefinite representations of material failure criteria, Journal of Optimization Theory and Applications 134 (2) (2007) 275-301.

[26] C. D. Bisbos, Semidefinite optimization models for limit and shakedown analysis problems involving matrix spreads, Optimization Letters 1 (1) (2007) 101-109.

[27] J. Salençon, Calcul à la rupture et analyse limite, Presses de l'Ecole Nationale des Ponts et Chaussées, 1983.

[28] A. p. s. MOSEK, The MOSEK optimization software, online at http://www. mosek. com, 2010.

[29] A. H. Cheng, On generalized plane strain poroelasticity, International Journal of Rock Mechanics and Mining Sciences 35 (2) (1998) 183-193.

[30] I. Ahmadi, M. M. Aghdam, Generalized Plane strain Meshless Local Petrov-Galerkin method for Micromechanics of Thermo-mechanical loading of composites, Journal of Mechanics of Materials and Structures 5 (4) (2010) 549-566.

[31] S. Maghous, A numerical approach to the yield strength properties of periodic heterogeneous materials (in French), Ph.D. thesis, ENPC (Paris), 1991. 